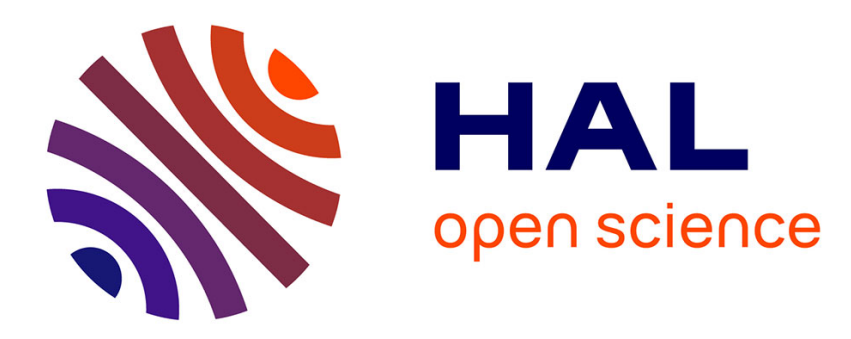

\title{
Les tirailleurs, bras armé de la France coloniale Emmanuel Blanchard
}

\section{To cite this version:}

Emmanuel Blanchard. Les tirailleurs, bras armé de la France coloniale. Plein Droit, 2003, 56, http://www.gisti.org/doc/plein-droit/56/tirailleurs.html. hal-00746477

\section{HAL Id: hal-00746477 \\ https://hal.science/hal-00746477}

Submitted on 29 Oct 2012

HAL is a multi-disciplinary open access archive for the deposit and dissemination of scientific research documents, whether they are published or not. The documents may come from teaching and research institutions in France or abroad, or from public or private research centers.
L'archive ouverte pluridisciplinaire HAL, est destinée au dépôt et à la diffusion de documents scientifiques de niveau recherche, publiés ou non, émanant des établissements d'enseignement et de recherche français ou étrangers, des laboratoires publics ou privés. 


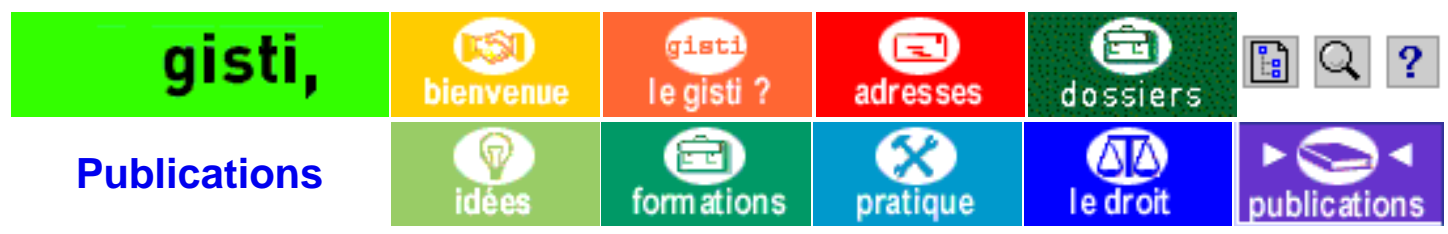

Q Plein Droit
$\square$ Numéros
$\square$ Index
$\checkmark$ En ligne
Cahiers
Notes juridiques
Guides
Notes pratiques
Hors-collection
Commandes
Archives
Photocopillage

Plein Droit n ${ }^{\circ}$ 56, mars 2003

«Les spoliés de la décolonisation »

\section{Les tirailleurs, bras armé de la France coloniale}

\author{
Emmanuel Blanchard \\ Enseignant en sciences économiques et sociales
}

Longtemps occultée, la participation des populations coloniales aux efforts de guerre de la France est aujourd'hui un véritable enjeu de mémoire au cœur des luttes politiques et juridiques des anciens combattants et des sans-papiers. En mettant l'accent sur la contribution de leurs aînés à la défense d'idéaux démocratiques, mis à mal par les gouvernements passés et présents de la France des colonies ou de la fermeture des frontières, ces derniers ont contribué à sortir de l'oubli des milliers d'hommes dont les sacrifices ne sont toujours pas reconnus. Il reste que l'image du tirailleur libérateur de la France occupée ne permet pas d'appréhender, dans toute sa complexité, l'histoire des troupes coloniales.

C'est dès le XVIle siècle que fut créée « la coloniale » chargée de défendre les vieilles colonies du royaume de France par des recrutements locaux afin de compléter ses « unités blanches ». Mais c'est avec la fondation du second empire colonial, au cours du XIXe siècle, que les « troupes indigènes » vont prendre une véritable importance quantitative et qualitative au sein de l'armée française.

La longue et meurtrière conquête de l'Algérie entamée en 1830 donna lieu, dès 1832, à la création de bataillons de "Turcos », fondés sur les décombres des milices ottomanes vaincues, qui seront la souche des «bataillons de tirailleurs indigènes " (1842) puis des « régiments de tirailleurs algériens » (1856). Cet exemple fut ensuite suivi en Afrique du Nord (tirailleurs tunisiens puis marocains) et de l'Ouest, avec la formation, par le général Faidherbe, dès 1857, des premiers bataillons de tirailleurs sénégalais. Ils prenaient la succession de troupes auxiliaires formées d'esclaves rachetés par l'armée française et, sous cette appellation générique, accueillirent l'ensemble des populations conquises au fur et à mesure de l'avancée de l'armée d'Afrique dans les territoires de la future Afrique occidentale française (AOF).

L'utilisation première de ces troupes fut donc bien de mener la politique de conquête et de pacification de nouvelles régions destinées à intégrer l'empire colonial français. Très vite cependant, du fait de l'efficacité et de l'ardeur au combat de certaines d'entre elles, elles furent utilisées loin de leurs bases et engagées dans les aventures extérieures du Second Empire (guerre de Crimée, 1854-56 ; intervention au Mexique, 
1862-1867). Surtout, quelques bataillons furent appelés au front lors de la guerre de 1870-71. De cette époque et du traumatisme de la défaite de Sedan date d'ailleurs une nouvelle vision du potentiel de ces régiments et des territoires dont ils provenaient.

Sous l'impulsion d'officiers de l'armée d'Afrique, ces régions difficilement conquises et pacifiées acquièrent dans l'esprit d'une partie du personnel politique, jusqu'alors relativement indifférent, une importance stratégique. Cette Force noire [1] fut de plus en plus envisagée comme une "réserve d'hommes » qui permettait enfin de lutter d'égal à égal contre l'ogre démographique allemand.

Contre l'avis même des colons, peu désireux de voir les indigènes armés par la puissance contre laquelle ils se soulevaient périodiquement, de nouveaux bataillons furent formés et une conscription partielle fut même introduite en Algérie (1896) et dans les villes libres du Sénégal (Saint-Louis, Dakar...). Ce passage dans l'armée s'accompagna, pour les anciens conscrits, d'avantages (emplois ou terrains réservés) et d'évolutions de leur statut (possibilité de ne plus être soumis au code de l'indigénat) qui furent, toutefois, le plus souvent accordés sur le mode de la faveur et non du droit.

Cette place particulière des anciens combattants dans les sociétés colonisées restera une constante : leur passage progressif de privilégiés, ardents défenseurs d'une France leur faisant miroiter la marche progressive vers l'égalité de droits, à celui de francophiles aux espoirs déçus, a joué un rôle dans le basculement, quelques décennies plus tard, de certaines régions dans la lutte armée pour l'indépendance. A cet égard, les deux guerres mondiales ont d'ailleurs été des moments clés...

La mobilisation des troupes coloniales pour la guerre 1914-1918 fut sans précédent : environ 800000 hommes ont été incorporés, plus de 70000 y perdirent la vie [2]. Lors de la bataille des Dardanelles, les « tirailleurs sénégalais » représentaient, à eux seuls, la moitié des effectifs engagés. Cette mobilisation ne s'est pas faite sans difficultés, la solde et les avantages traditionnels n'étant plus suffisamment convaincants. Le premier député du Sénégal (de la ville libre de Saint-Louis), Blaise Diagne fut appelé à la rescousse au début de l'année 1918 pour convaincre ses électeurs, et se prononça pour une généralisation de la conscription qui, dans son esprit, devait s'accompagner d'une marche progressive vers la citoyenneté : à l'égalité dans les tranchées et devant la mort devait correspondre celle dans la société. Ses arguments ne suffirent cependant pas à convaincre les réfractaires au départ sur les champs de bataille européens.

En effet, depuis de longs mois, l'intérieur de l'AOF était secoué par des révoltes régulières contre les enrôlements forcés. Ces émeutes, d'ailleurs réprimées par les tirailleurs restés sur place, prirent une telle ampleur, que le gouverneur de l'AOF dût suspendre un temps les recrutements militaires et suggérer au gouvernement de mettre l'accent sur la contribution économique des colonies. Au renforcement des exportations 
vers la métropole, s'ajouta donc une contribution importante en termes de main-d'œuvre. L'exemple des travailleurs chinois est maintenant bien connu [3], mais ce sont plus de 200000 travailleurs coloniaux (dont plus de 50000 Indochinois) qui vinrent assurer la relève des conscrits dans les usines françaises.

\section{«Nous ne sommes plus que des nègres "}

L'horreur des combats et la peur de la mort en moins, leur statut n'était guère éloigné de celui de leurs compatriotes soldats puisque, eux aussi, étaient soumis à un statut militaire et vivaient dans des casernements surveillés par l'armée. Pour tous ces hommes, se posa, en 1918, la question du retour, le gouvernement ne tenant absolument pas à les voir s'installer sur le territoire français. Cette découverte de la métropole incita cependant certains d'entre eux à fuir les rapatriements et à s'installer définitivement ou provisoirement en France.

Pour les autres, le retour fut souvent synonyme de désillusions puisque les maigres pensions de combattants auxquelles certains avaient droit ne leur étaient de fait pas versées, et que la citoyenneté pour tous n'était toujours pas à l'ordre du jour : "lorsqu'on a besoin de nous pour nous faire tuer ou nous faire travailler, nous sommes des Français ; mais quand il s'agit de nous donner des droits, nous ne sommes plus des Français, nous sommes des nègres " [4]. A cette époque, seules la fierté d'avoir contribué à la victoire militaire et l'admiration et la peur de la puissance militaire française empêchèrent ces anciens combattants de rallier massivement les mouvements nationalistes qui, dans l'entre-deux guerres, émergeaient dans de nombreuses colonies.

La propagande des Mangin et consorts en faveur de la Force noire n'ayant eu qu'un succès limité, l'engagement des troupes coloniales, au cours de la première guerre mondiale, fut progressif et quantitativement faible (les bataillons coloniaux représentaient moins de $5 \%$ de l'ensemble des troupes engagées dans les combats). Ce n'est qu'au fur et à mesure des batailles que le professionnalisme et la bravoure de ces troupes furent reconnus. Elles arrivèrent d'ailleurs en métropole peu expérimentées et mal préparées. De nombreux bataillons restèrent ainsi en réserve et l'inadaptation climatique et la maladie tuèrent au moins autant que les combats.

Lors de la seconde guerre mondiale, la situation fut tout autre : les troupes coloniales furent d'emblée massivement intégrées aux plans de bataille et, placées en première ligne, elles payèrent un très lourd tribut lors des combats de mai et juin 1940 [5]. Avec la défaite, les nombreux prisonniers furent enfermés dans des camps de travail au service de l'effort de guerre allemand, en métropole ou outre-Rhin. Ils furent la cible d'une intense propagande de la part des services allemands qui essayaient de s'appuyer sur les sentiments nationalistes des originaires d'Afrique du Nord notamment.

A la Libération, tant dans les départements algériens qu'en AOF, les autorités locales mirent en garde Paris sur les risques 
politiques liés au rapatriement de ces prisonniers " retournés 》 par l'Allemagne ou, en tout cas, facilement enclins à alimenter la contestation sociale et politique. Les désarmements, contrôles d'identité et d'états de service préalables au retour donnèrent lieu à de multiples incidents, les soldats coloniaux supportant mal que leur contribution patriotique et militaire soit l'objet de suspicion. Nombre d'entre eux étaient en effet membres de l'armée d'Afrique à partir de laquelle la France libre se lança dans la reconquête du territoire national. En 1944, ils représentaient ainsi la moitié des troupes ayant débarqué en Provence. Ces troupes furent cependant "blanchies » [6] au fur et à mesure de leurs avancées : de Gaulle privilégiant l'intégration des groupes de résistants à la 1 re armée, il choisit, face à la pénurie de moyens, de désarmer une partie des bataillons de tirailleurs afin d'équiper ces nouveaux combattants.

La frustration de se voir déposséder d'un rôle central, les suspicions des autorités françaises déjà évoquées et surtout les promesses matérielles non tenues furent à l'origine du soulèvement du camp de Thiaroye (banlieue de Dakar). Le 1 er décembre 1944, les troupes françaises ouvrirent le feu sur 1280 tirailleurs en cours de rapatriement. Le lourd bilan (35 tués, 35 blessés graves...) suffit, à lui seul, à démontrer la complexité du rôle et de l'état d'esprit des troupes coloniales à la Libération : fêtées en métropole par la population en liesse mais réprimées et ayant toutes les difficultés pour faire valoir leurs droits dès lors qu'elles réintégraient leurs régions d'origine.

Le racisme des sociétés coloniales est d'ailleurs souvent opposé à la fraternité au sein des unités combattantes. II est vrai que, pour beaucoup d'engagés coloniaux, cette voie était aussi un moyen d'échapper aux discriminations et charges auxquelles les assujettissaient le code de l'indigénat et autres législations d'exception. De plus, quand ils avaient l'occasion de servir en métropole, nombreux étaient ceux qui remarquaient que la population locale était moins xénophobe que les colons. II n'en reste pas moins que, faisant preuve d'un paternalisme indéniable, l'armée française était loin d'être égalitaire. Avancement lent et bloqué, impossibilité de commander des troupes non indigènes, placement sous les ordres de métropolitains moins gradés étaient le lot commun des rares officiers africains.

Ces discriminations et ségrégations (mess séparé, voyage sur des bateaux différents...) ont perduré, sinon légalement, du moins dans les faits, à la Libération. Elles expliquent en partie le ralliement progressif d'une partie des anciens combattants coloniaux à la cause nationaliste et le passage à l'ennemi lors des guerres d'Indochine et surtout d'Algérie de nombreux cadres de l'armée française. Ces ralliements ne furent cependant pas systématiques et eurent lieu le plus souvent tardivement ; la plupart des officiers algériens ralliés à l'Armée de libération nationale avaient ainsi combattu en Indochine et ne rejoignirent l'ALN qu'en 1957-1958.

Dès la fin de la seconde guerre mondiale, les bataillons de tirailleurs furent en effet massivement utilisés lors des multiples 
conflits coloniaux (en Indochine surtout, où près d'un combattant sur deux était originaire des colonies, mais aussi lors de l'insurrection de 1947 à Madagascar ou, à plusieurs reprises, en Tunisie et au Maroc). Le commandement français se faisait fort d'ailleurs de manipuler les ressentiments et oppositions entre les différentes populations coloniales pour mieux maintenir l'ordre.

Même si des bataillons de Marocains et de Tunisiens refusèrent d'aller servir en Algérie au début des " événements », ou qu'au cours de cette guerre, la méfiance sur la fidélité des troupes coloniales ne cessa de grandir, cette période de l'après seconde guerre mondiale est aussi celle où les troupes coloniales se professionnalisent vraiment et voient leurs modes d'engagement se multiplier (elles sont ainsi utilisées pour le maintien de l'ordre en métropole, notamment en 1948 lors de la grande grève des mineurs). Le nombre de ceux qui obtinrent alors le droit à une pension de retraite, et non pas à la seule retraite du combattant, augmenta donc fortement [7].

Ce constat et la perpétuation de logiques discriminatoires ancrées dans l'histoire de ces troupes conduiront à la cristallisation des pensions à partir de 1958.

\section{Les Chinois de 14-18}

Quatorze juillet 2000, huit heures du matin, place de la République : une petite foule bruyante de soixante sans-papiers chinois, accompagnés de quelques amis français, embarquent à bord d'un car qui prend aussitôt le chemin du Nord. Ces sans-papiers appartiennent au Troisième collectif et ont décidé d'aller rendre hommage à leurs compatriotes tombés au service de la France pendant la guerre de 1914-1918.

Ce sont en effet quelque 140000 ressortissants chinois qui ont été amenés en France par les gouvernements français et anglais pour travailler soit dans les usines de munitions, soit dans les chantiers de creusement ou de remblaiement des tranchées, soit encore au déminage. Appâtés par des promesses qui ne seront pas toujours tenues, ils sont traités très durement ; la promiscuité, le froid, le manque d'hygiène font des ravages parmi eux ; sur le trajet qui conduit des camps où ils sont parqués à leur lieu de travail, ils sont souvent insultés par la population. Plusieurs milliers d'entre eux y laisseront leur vie. Quelque huit cents de ces victimes sont inhumées au cimetière de Noyelles-sur-Mer, à proximité d'Abbeville, et c'est dans ce cimetière, entretenu par le gouvernement britannique, que les sans-papiers du Troisième collectif se rendent en ce 14 juillet 2000.

Accueillie par la secrétaire de la section de la Ligue des droits de l'homme d'Abbeville, la délégation parcourt silencieusement les allées, afin de lire et de relire les épitaphes gravées sur les stèles. Puis une gerbe est déposée, portant une inscription ainsi rédigée : 


\section{Aux travailleurs chinois de 1917-1920 morts pour la France, \\ Les travailleurs chinois de 1997-2000 rejetés par la France.}

Après quelques brèves interventions rappelant l'histoire du site et la situation actuelle, après une copieuse séance de photos, les voyageurs se dispersent quelques instants aux abords du cimetière. Puis c'est l'heure du retour ; la délégation revient à Paris avec le sentiment qu'un peu de justice a été rendue.

\section{Pour en savoir plus}

Clayton Antony, Histoire de l'armée française en Afrique 1830-1962, Albin Michel, 1994.

Recham Belkhacem, Les musulmans algériens dans l'armée française (1919-1962), l'Harmattan, 1996.

Kamian Bakari, Des tranchées de Verdun à l'église SaintBernard. 80000 combattants maliens au service de la France(1914-1918 et 1939-1945), Karthala, 2001.

Michel Marc, L'appel à l'Afrique. Contributions et réactions à l'effort de guerre en AOF (1914-1919), publications de la Sorbonne, 1982.

Echenberg Myron, Colonial conscripts. The tirailleurs sénégalais in French West Africa 1857-1960, Portsmouth, Heinemann, London, James Currey, 1991.

\section{Notes}

[1] Le général Mangin, auteur de la Force noire (1910) est de ceux qui ont beaucoup fait pour la diffusion de cette idée et la catégorisation des combattants dans une perspective culturaliste voire raciste.

[2] Les pertes françaises totales sont de 1,3 million d'hommes sur plus de 5 millions de combattants. Cette proportion de décès est la même dans les troupes coloniales engagées sur le front.

[3] Yu-Sion Live, "Les travailleurs chinois et l'effort de guerre ", Hommes et migrations, $\mathrm{n}^{\circ} 1148$, novembre 1991, pp.12-15. Voir aussi la section «Les Chinois de 14-18».

[4] Senghor Lamine (aucun lien de parenté avec le poète) dans la revue La voix des nègres, mars 1927, cité par Dewitte Philippe, «La dette du sang ", Hommes et migrations, novembre 1991, n 1148, pp.8-11.

[5] Environ $40 \%$ des soldats d'AOF et d'AEF engagés en 1939-1940 trouvèrent la mort alors que le taux de mortalité des combattants "Français de France » ne fut que de $3 \%$.

[6] L'expression était couramment utilisée pour désigner l'augmentation de la proportion de métropolitains dans les troupes coloniales. 
[7] Pour une définition des différents types de pensions, voir, dans ce numéro, l'encadré p. 11.

Dernière mise à jour : 13-03-2003 20:14

Cette page : http://www.gisti.org/ doc/plein-droit56/tirailleurs.html

Bienvenue | Le Gisti? |Adresses | Idées | Formations | Pratique | Le droit | Publications

Page d'accueil | Recherche | Plan du site | Aider le Gisti | Autres sites

Comment contacter le Gisti 